\title{
ESQUEMAS MALADAPTATIVOS TEMPRANOS Y AGRESIVIDAD EN ESTUDIANTES DE SECUNDARIA DE LIMA METROPOLITANA
}

\author{
Lizbeth Pamela Collado Osorio \\ https://orcid.org/0000-0002-7696-0847
}

\author{
María Matalinares Calvet \\ https://orcid.org/0000-0003-1052-6922
}

Universidad Nacional Mayor de San Marcos, Lima, Perú

Recibido: 10 de marzo del 2019 / Aceptado: 14 de julio del 2019

doi: 10.26439/persona2019.n022(2).4564

\begin{abstract}
Resumen. La presente investigación tuvo como objetivo establecer la relación que existe entre los esquemas maladaptativos tempranos y la agresividad. La muestra estuvo conformada por 641 estudiantes de cuarto y quinto año de secundaria de Lima Metropolitana, entre los 14 y 18 años de edad. El diseño del estudio fue descriptivo correlacional y comparativo, y los instrumentos empleados fueron el cuestionario de agresión de Buss y Perry (1992) y el cuestionario de esquemas desadaptativos tempranos (YSQ-L2) de Young (1990). Los resultados demuestran que existe una relación significativa y positiva entre los esquemas maladaptativos tempranos y la agresividad. En el plano descriptivo, los esquemas que prevalecieron fueron privación emocional e insuficiente autocontrol / autodisciplina; en la escala de agresividad y en las subescalas predominó el nivel medio, con excepción del componente ira, que obtuvo una puntuación baja.
\end{abstract}

Palabras clave: esquemas / maladaptación / agresividad / estudiantes de secundaria

\section{EARLY MALADAPTIVE SCHEMAS AND AGGRESSIVENESS IN HIGH SCHOOL STUDENTS FROM LIMA METROPOLITAN AREA}

\begin{abstract}
This research aimed to establish the relationship between early maladaptive schemas and aggressiveness. The sample consisted of 641 fourth- and fifth-year high school students aged 14 to 18 from Lima Metropolitan Area. The study had a descriptive, correlational and comparative design. The Buss-Perry Aggression Questionnaire (1992) and the Young Schema Questionnaire Long Form - Second Edition (YSQ-L2) were used in the study. The results show a significant and positive relationship between early maladaptive schemas and aggressiveness. At the descriptive level, the most prevalent schemas were emotional deprivation and insufficient self-control/self-discipline. On the aggressiveness scale and subscales, the results showed average levels, with the exception of the anger component, which had a low score.
\end{abstract}

Keywords: schemas / maladaptation / aggressiveness / high school students 


\section{INTRODUCCIÓN}

El ser humano es un ser social que, desde el momento de su concepción, se encuentra en constante interacción con el medio que lo rodea. Este proceso abre la adquisición y desarrollo de creencias, costumbres, valores y conductas (Orcacita y Uribe, 2010).

Según el Instituto Nacional de Estadística e Informática, durante el trimestre eneromarzo del 2019 se identificó que el 52 \% de hogares nacionales tiene miembros menores de dieciocho años; es decir, en las familias peruanas existe un elevado número de niños, niñas y adolescentes. A partir de ello se desprende la importancia de promover, desde temprana edad, un desarrollo biopsicosocial saludable.

Si durante los primeros años de vida no se llega a cubrir satisfactoriamente las necesidades emocionales del infante $y$, por el contrario, se vivencian experiencias tempranas disfuncionales, es altamente probable que la persona adquiera, desarrolle y elabore a lo largo de su vida esquemas maladaptativos tempranos (Rodríguez, 2009).

Los esquemas maladaptativos tempranos son pensamientos y emociones disfuncionales que poseen la característica de ser estables y duraderos. Se desarrollan a partir de experiencias no saludables con personas significativas durante los primeros años de vida (Young, 1999, citado por Castrillón et al., 2005).

Es por ello fundamental ofrecer una niñez saludable, seguida de un acompañamiento socioemocional adecuado durante la adolescencia, ya que si el adolescente no posee factores protectores puede convertirse en una persona altamente vulnerable a los factores de riesgo.

La adolescencia es un periodo de desarrollo entre la niñez y la vida adulta. Es necesario vivir esta etapa, puesto que nos permitirá realizarnos como seres humanos maduros, responsables y creativos (Rice, 2000). Erickson (1968, citado por Rice, 2000) expuso que la adolescencia es una crisis normativa, una fase normal en la cual la percepción de los conflictos aumenta. El adolescente continúa la búsqueda de su identidad personal durante un periodo de cambio social y constante exposición a situaciones de peligro.

Los adolescentes emocionalmente inestables y con un débil soporte sociofamiliar tienden a expresar sus emociones a través de conductas disfuncionales tales como fugas del hogar, adicción y agresividad, entre otros. En un estudio realizado por Matalinares, Arenas, Díaz y Dioses (2014) con una muestra representativa de estudiantes adolescentes de las tres regiones del Perú (costa, sierra y selva), se identificó que existe una relación positiva y altamente significativa entre la adicción y la agresividad. Ante estos resultados, se resaltó la importancia de realizar actividades preventivas con los estudiantes y padres. 
De lo planteado se desprende el objetivo de la presente investigación, que pretende identificar los pensamientos y emociones disfuncionales del adolescente, adquiridos a temprana edad y desarrollados durante su proceso de socialización, y correlacionarlos con conductas de riesgo vinculadas con el escaso control de las emociones y la búsqueda, impulsiva o premeditada, de causar daño a los demás. En otras palabras, este estudio busca determinar la relación entre los esquemas maladaptativos tempranos y la agresividad en estudiantes de secundaria de Lima Metropolitana.

La agresividad, según lo planteado por Buss (1961, citado por Serrano, 2003) es la respuesta caracterizada por ser un estímulo nocivo para los demás; a su vez, Coie y Dodge (1998, citados por Shaffer y Kipp, 2007) exponen que el acto agresivo es cualquier conducta que busca lastimar o perjudicar a otro que se siente motivado a evitarlo. Puede añadirse a ello lo expuesto por Bandura (1973, citado por Rice, 2000), quien señala que el aprendizaje social ejerce gran influencia en la conducta humana; por ejemplo, observar que otros son recompensados por la emisión de una conducta agresiva aumenta la posibilidad de que el observador también ejerza agresión; es decir, los factores sociales y ambientales guardan estrecha relación con la conducta aprendida y moldeada del adolescente.

La agresividad ha sido estudiada por diversos autores, dentro de los cuales se identifica a Inglés et al. (2014), quien, en una muestra de 314 estudiantes adolescentes en España, encontró que la agresividad se encuentra correlacionada negativamente con la inteligencia emocional. Por su parte, Andreu, Peña y Penado (2013) hallaron que los adolescentes agresivos presentan mayores niveles de impulsividad que los adolescentes no agresivos.

En el Perú, Quijano y Ríos (2015), al igual que Carbajal y Jaramillo (2015) y Luna (2017), encontraron niveles medios de agresividad en estudiantes de secundaria. Estos resultados -reforzados por Molina (2018) y Agip (2018), quienes también hallaron niveles altos de agresividad en muestras similares- exponen dificultades en el adolescente para ejercer control sobre sus pensamientos y emociones. Asimismo, diversos autores muestran la importante influencia que ejerce el ambiente sociofamiliar sobre la conducta del ser humano. Por ejemplo, Arias (2013) halló que cuando el vínculo entre el adolescente y las figuras significativas dentro del hogar tiende a debilitarse, las conductas agresivas suelen manifestarse; por su parte, Matalinares et al. (2010) y Arangoitia (2017) demostraron que existe una relación significativa e inversa entre clima familiar y agresividad; es decir, ante un ambiente disfuncional familiar los niveles de agresividad tienden a aumentar.

Ante lo mencionado, se resalta la importancia de adquirir y desarrollar pensamientos y emociones funcionales durante la formación del carácter. Chupillón (2019) identificó que los adolescentes que experimentan carencia de afecto, inestabilidad 
emocional y falta de disciplina a temprana edad tienden a desarrollar conductas agresivas premeditadas e impulsivas, y Salvatierra (2017) halló una correlación directa y significativa entre las variables esquemas maladaptativos tempranos y agresividad. Asimismo, aquellos adolescentes con problemas de conducta suelen desarrollar esquemas maladaptativos tempranos tales como deprivación emocional, insuficiente autocontrol/autodisciplina, desconfianza/abuso (Flores y Vilca, 2017). Resultados similares se obtuvieron en una muestra representativa de madres adolescentes, en quienes predominaron los esquemas abandono, privación emocional, desconfianza/abuso; es decir, a temprana edad, estas jóvenes adquirieron la creencia de que las personas más significativas de su entorno, no podrían brindarles el apoyo y estabilidad necesario para conseguir cubrir sus necesidades afectivas (Pérez y Torres, 2016).

En el ámbito internacional, Sánchez, Andrade y Lucio (2019) identificaron que los esquemas maladaptativos tempranos se relacionan positiva y significativamente con la ansiedad. Por su parte, Gantiva, Bello, Vanegas y Sastoque (2009) expusieron la presencia de una correlación significativa entre la historia de maltrato físico desarrollada en la infancia y los esquemas de abandono y derecho perpetuados posteriormente.

Añadido a lo expuesto, se resalta que los resultados del presente trabajo permiten conocer más sobre el adolescente y contribuir, a partir de ello, a la formación de programas preventivos promocionales. De igual forma, la información adquirida permitirá al contexto sociofamiliar del menor tomar conciencia acerca de lo importante y significativo que es ejecutar un desarrollo socioemocional saludable en el niño, infante y adolescente.

En la actualidad, la presencia de esquemas maladaptativos tempranos y agresividad en adolescentes deben seguir explorándose, pues hay pocos estudios que investiguen dichas variables. Además, esta investigación muestra un aporte, tanto teórico como práctico, que puede contribuir al desarrollo de futuras investigaciones.

\section{Hipótesis}

Hipótesis general

$H_{1}$ : Existe relación estadísticamente significativa entre los esquemas maladaptativos tempranos y la agresividad en estudiantes de secundaria de Lima Metropolitana.

Hipótesis específicas

$H_{1.1}$ : Existe relación estadísticamente significativa entre los esquemas maladaptativos tempranos y los factores de la agresividad en estudiantes de secundaria de Lima Metropolitana.

$H_{1.2}$ : Existen diferencias estadísticamente significativas entre los esquemas maladaptativos tempranos y los factores de la agresividad en estudiantes de secundaria de Lima Metropolitana, según sexo y tipo de institución. 


\section{MÉTODO}

En el desarrollo del estudio, con relación a las hipótesis planteadas se utilizó en un primer momento el diseño descriptivo correlacional con el objetivo de relacionar las dos variables; posteriormente, se compararon los resultados obtenidos por los estudiantes según sexo y tipo de institución. Para ello, se empleó el diseño descriptivo comparativo (Sánchez y Reyes, 2009).

\section{Participantes}

La población de estudio estuvo formada por 29120 estudiantes de cuarto y quinto de secundaria de Lima Metropolitana (Ministerio de Educación [Minedu], 2018). El tipo de muestreo fue intencional (Salgado, 2018), en tanto que los criterios de inclusión fueron: estudiantes de catorce a dieciocho años, tanto de sexo masculino como femenino, pertenecientes a instituciones particulares y estatales de Lima Metropolitana.

La muestra de estudio estuvo compuesta por 641 estudiantes de cuarto y quinto año de secundaria de cuatro distritos de Lima Metropolitana (Miraflores, Santiago de Surco, Puente Piedra y Comas), de los cuales el $51,3 \%$ eran mujeres y el $48,7 \%$ varones. En lo que respecta al grado académico, la mayor parte de la muestra cursaba el quinto año (66,4 \%); a su vez, las instituciones educativas de gestión pública agruparon al 66,1% de estudiantes.

Tabla 1

Características sociodemográficas en una muestra de estudiantes de nivel secundario ( $N=641)$

\begin{tabular}{lrrllc}
\hline & $\mathrm{N}$ & $\%$ & & $\mathrm{~N}$ & $\%$ \\
\hline Edad (años) & & & Grado & \\
\hline 14 & 17 & 2,7 & Cuarto & 215 & 33,5 \\
15 & 187 & 29,2 & Quinto & 426 & 66,5 \\
16 & 334 & 52,1 & & & \\
17 & 88 & 13,7 & & & \\
18 & 15 & 2,3 & & & \\
\hline Sexo & & & Gestión escolar & \\
\hline Mujer & 329 & 51,3 & Público & 424 & 66,1 \\
Varón & 312 & 48,7 & Privada & 217 & 33,9 \\
\hline Total & 641 & 100,0 & Total & 641 & 100,0 \\
\hline
\end{tabular}

Nota: $\mathrm{N}$ = número de casos. $\%$ = porcentaje 


\section{Instrumentos}

Cuestionario de esquemas desadaptativos tempranos (YSQ-L2), cuyos autores son Jeffrey E. Young y Gary Brown (1990). Esta prueba fue validada por Castrillón et al. (2005) en una muestra de estudiantes universitarios en Medellín, Colombia, y fue estandarizado por León y Sucari (2013) para la realidad nacional. La prueba tiene como objetivo evaluar once esquemas disfuncionalmente adquiridos a temprana edad. Su administración puede ser individual o colectiva, en tanto que el tiempo de aplicación fluctúa entre los treinta y cuarenta minutos. Consta de 45 ítems que se puntúan según la escala tipo Likert de seis valores.

La confiabilidad fue establecida mediante el método de consistencia interna con el alfa de Cronbach ordinal (Gandermann, Guhn y Zumbo, 2012; Domínguez-Lara, 2018), que osciló entre .45 y .87. A su vez, cada estimación del alfa de Cronbach ordinal es acompañada por sus intervalos de confianza al $95 \%$ siguiendo la propuesta de Fisher (Romano, Kromrey, Owens y Scott, 2011; Turner, 2015).

Tabla 2

Confiabilidad por consistencia interna de cuestionario de esquemas de Young para adolescentes

\begin{tabular}{lcc}
\hline Variable y dimensiones & Número de ítems & Alfa de Cronbach ordinal ${ }^{(\mathrm{a})}$ \\
\hline Abandono & 6 & .87 \\
Insuficiente control/autodisciplina & 6 & $.850-.888]$ \\
Desconfianza/abuso & 5 & .84 \\
Privación emocional & 5 & $.74-.861]$ \\
Vulnerabilidad & & {$[.703-.773]$} \\
Autosacrificio & 4 & .77 \\
Estándares inflexibles 1 & 4 & {$[.736-.800]$} \\
Estándares inflexibles 2 & & .87 \\
Inhibición emocional & 4 & {$[.850-.888]$} \\
Derecho & 3 & .77 \\
Entrampamiento & 3 & $.736-.800]$ \\
\hline
\end{tabular}

Nota: (a) Este método fue seleccionado por las alternativas de respuesta tipo Likert. 
El cuestionario de agresión de Buss y Perry (1992), adaptado por Matalinares et al. (2012), evalúa cuatro dimensiones (agresión física, agresión verbal, hostilidad e ira). La prueba consta de veintinueve ítems, cuya aplicación puede ser individual o colectiva. Su tiempo de administración es de aproximadamente veinte minutos.

La confiabilidad fue estimada con el alfa de Cronbach ordinal (Gandermann, Guhn y Zumbo, 2012; Domínguez-Lara, 2018) y fue de .92, nivel considerado elevado por Murphy y Davishofer (1998, citado en Hogan, 2015). Con respecto a las dimensiones, estas lograron un alfa de Cronbach ordinales moderado: .79 y .84 (Murphy y Davishofer, 1998, citado en Hogan, 2015). Asimismo, cada estimación del alfa de Cronbach ordinal es acompañada por sus intervalos de confianza al 95 por ciento, siguiendo la propuesta de Fisher (Romano, Kromrey, Owens y Scott, 2011; Turner, 2015).

Tabla 3

Confiabilidad por consistencia interna del cuestionario de agresión de Buss y Perry

\begin{tabular}{lcc}
\hline Variable y dimensiones & Número de ítems & Alfa de Cronbach ordinal $^{\left({ }^{(a)}\right.}$ \\
\hline Agresividad & 29 & .92 \\
Agresividad física & 9 & {$[.907-.931]$} \\
Agresividad verbal & 5 & .84 \\
Hostilidad & 8 & $.816-.861]$ \\
Ira & 8 & {$[.759-.817]$} \\
& 7 & .79 \\
\end{tabular}

Nota: (a) Este método fue seleccionado por las alternativas de respuesta tipo Likert.

\section{Procedimiento}

La recolección de datos se llevó a cabo de forma colectiva en cuatro colegios pertenecientes a diferentes distritos de Lima Metropolitana (Miraflores, Santiago de Surco, Puente Piedra y Comas). Para ello, inicialmente se realizaron las coordinaciones correspondientes con las autoridades de las instituciones; seguidamente, se informó a los estudiantes acerca de los objetivos de la investigación y se hizo entrega de los consentimientos informados (firmados por los padres de familia). Los instrumentos se aplicaron en el horario de tutoría; antes de ello, se remarcó a los estudiantes que los datos que brindaban se emplearían únicamente para fines académicos. Asimismo, se respetó el anonimato de los participantes y el derecho de no responder si así lo decidían. 


\section{Análisis de datos}

La información recabada se trasladó a una plantilla del programa Excel versión 2013; posteriormente, se exportó al programa estadístico SPSS versión 25. En el programa SPSS fueron codificadas las variables de estudio para su correcta identificación y posterior análisis.

En cuanto al aspecto descriptivo, se analizaron las características sociodemográficas de la muestra y las variables objetivo de estudio (para lo cual se usaron los baremos locales de cada una). Los resultados se presentan en tablas de frecuencias y porcentajes. El análisis inferencial fue realizado mediante pruebas no paramétricas debido a la ausencia de un muestreo probabilístico que garantizara la representatividad de la muestra (Manterola y Otzen, 2015). La prueba estadística utilizada fue rho de Spearman.

\section{RESULTADOS}

Esta sección contiene el procesamiento de la información recopilada; para ello, se han seguido las hipótesis planteadas por el estudio. Previamente se presentan los descriptivos de las variables.

\section{Análisis descriptivo}

Los esquemas maladaptativos tempranos considerados con un nivel significativamente disfuncional fueron aquellos que lograron un puntaje ubicado por encima del percentil 85 ( $\geq$ Pc 85 ). Según la tabla 4, los esquemas maladaptativos con mayores porcentajes dentro del nivel disfuncional fueron: privación emocional $(38,2 \%)$ e insuficiente autocontrol/autodisciplina (16,2\%); en contraste, los esquemas maladaptativos tempranos con menores porcentajes dentro del nivel disfuncional fueron: inhibición emocional (6,2 \%) y estándares inflexibles 2 (6,9\%). 
Tabla 4

Niveles significativos de esquemas maladaptativos tempranos en estudiantes de nivel secundario $(N=641)$

\begin{tabular}{|c|c|c|}
\hline & Frecuencia & Porcentaje \\
\hline \multicolumn{3}{|l|}{ Abandono $0^{(a)}$} \\
\hline Nivel significativo ( $\geq$ Pc 85) & 54 & 8,4 \\
\hline \multicolumn{3}{|c|}{ Insuficiente autocontrol/autodisciplina ${ }^{(a)}$} \\
\hline Nivel significativo ( $\geq$ Pc 85 ) & 104 & 16,2 \\
\hline \multicolumn{3}{|l|}{ Desconfianza/abuso ${ }^{(a)}$} \\
\hline Nivel significativo ( $\geq$ Pc 85) & 97 & 15,1 \\
\hline \multicolumn{3}{|l|}{ Privación emocional(a) } \\
\hline Nivel significativo ( $\geq$ Pc 85) & 245 & 38,2 \\
\hline \multicolumn{3}{|l|}{ Vulnerabilidad ${ }^{(a)}$} \\
\hline Nivel significativo ( $\geq$ Pc 85) & 66 & 10,3 \\
\hline \multicolumn{3}{|l|}{ Autosacrificio ${ }^{(a)}$} \\
\hline Nivel significativo ( $\geq$ Pc 85) & 63 & 9,8 \\
\hline \multicolumn{3}{|l|}{ Estándares inflexibles $1^{(a)}$} \\
\hline Nivel significativo ( $\geq$ Pc 85) & 91 & 14,2 \\
\hline \multicolumn{3}{|l|}{ Estándares inflexibles $2^{(a)}$} \\
\hline Nivel significativo ( $\geq$ Pc 85) & 44 & 6,9 \\
\hline \multicolumn{3}{|l|}{ Inhibición emocional(a) } \\
\hline Nivel significativo ( $\geq$ Pc 85) & 40 & 6,2 \\
\hline \multicolumn{3}{|l|}{ Derecho(a) } \\
\hline Nivel significativo ( $\geq$ Pc 85) & 76 & 11,9 \\
\hline \multicolumn{3}{|l|}{ Entrampamiento ${ }^{(a)}$} \\
\hline Nivel significativo ( $\geq$ Pc 85) & 47 & 7,3 \\
\hline Total & 641 & 100,0 \\
\hline
\end{tabular}

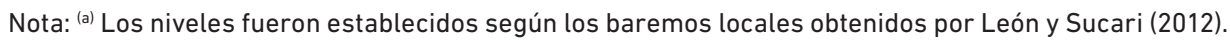

Con relación a la variable agresividad, en la muestra de estudio se observó un predominio del nivel medio $(33,9 \%$ y el $8,4 \%$ de los estudiantes se ubicó en un nivel muy alto (ver tabla 5). Dicha situación también estuvo presente en las dimensiones de la variable, excepto en ira, ya que en esta predominó el nivel bajo (30,3\%). Cabe señalar que el nivel muy alto estuvo representado por el 9,2 \% de agresividad física, 10,3\% de agresividad verbal, $7,3 \%$ de hostilidad y $7,2 \%$ de ira. 
Tabla 5

Niveles de agresividad y sus dimensiones en estudiantes de nivel secundario $(N=641)$

\begin{tabular}{|c|c|c|c|c|c|}
\hline & Frecuencia & Porcentaje & & Frecuencia & Porcentaje \\
\hline Agresividad(a) $^{(a)}$ & & & Hostilidad $^{(a)}$ & & \\
\hline Muy alto & 54 & 8,4 & Muy alto & 47 & 7,3 \\
\hline Alto & 151 & 23,6 & Alto & 165 & 25,7 \\
\hline Medio & 217 & 33,9 & Medio & 207 & 32,3 \\
\hline Bajo & 157 & 24,5 & Bajo & 162 & 25,3 \\
\hline Muy bajo & 62 & 9,6 & Muy bajo & 60 & 9,4 \\
\hline Agresividad física ${ }^{(a)}$ & & & $\operatorname{Ira} a^{(a)}$ & & \\
\hline Muy alto & 59 & 9,2 & Muy alto & 46 & 7,2 \\
\hline Alto & 131 & 20,4 & Alto & 152 & 23,7 \\
\hline Medio & 285 & 44,5 & Medio & 179 & 27,9 \\
\hline Bajo & 128 & 20,0 & Bajo & 194 & 30,3 \\
\hline Muy bajo & 38 & 5,9 & Muy bajo & 70 & 10,9 \\
\hline \multicolumn{6}{|l|}{ Agresividad verbal(a) } \\
\hline Muy alto & 66 & 10,3 & & & \\
\hline Alto & 172 & 26,8 & & & \\
\hline Medio & 186 & 29,1 & & & \\
\hline Bajo & 172 & 26,8 & & & \\
\hline Muy bajo & 45 & 7,0 & & & \\
\hline Total & 641 & 100,0 & Total & 641 & 100,0 \\
\hline
\end{tabular}

Nota: ${ }^{(a)}$ Los niveles fueron establecidos según los baremos locales obtenidos por Matalinares, Yaringaño, Uceda, Fernández, Huari, Campos y Villavicencio (2012).

\section{Análisis inferencial}

Este análisis fue desarrollado con pruebas no paramétricas, tal cual se ha indicado en la sección de análisis de datos. Se llevaron a cabo correlaciones estadísticamente significativas ( $p$-valor inferior al .05) entre los esquemas maladaptativos tempranos y agresividad. En la tabla 6 pueden verse correlaciones significativas entre los esquemas maladaptativos tempranos y las dimensiones de agresividad, con excepción de las siguientes correlaciones: autosacrificio y agresividad verbal, estándares inflexibles 2 y agresividad física, inhibición emocional y agresividad física, entrampamiento y agresividad física, entrampamiento y agresividad verbal.

Cabe mencionar que cada correlación estadística es acompañada de su tamaño del efecto $\left(r^{2}\right)$, los valores $r^{2}<.01$ son considerados insignificantes. 
Tabla 6

Correlación entre los esquemas maladaptativos tempranos y agresividad en estudiantes de nivel secundario $(N=641)$.

\begin{tabular}{|c|c|c|c|c|c|c|}
\hline Esquemas maladaptativos & & $A$ & AF & AV & I & $\mathrm{H}$ \\
\hline \multirow{3}{*}{ Abandono } & Rho Spearman & .335 & .125 & .174 & 424 & .346 \\
\hline & (p-valor) & $(.000)$ & $(.002)$ & $(.000)$ & $(.000)$ & $(.000)$ \\
\hline & $r^{2}$ & .126 & .016 & .031 & .180 & .120 \\
\hline \multirow{3}{*}{$\begin{array}{l}\text { Insuficiente control/ } \\
\text { autodisciplina }\end{array}$} & Rho Spearman & .441 & .286 & .318 & 418 & .414 \\
\hline & (p-valor) & $(.000)$ & $(.000)$ & $(.000)$ & $(.000)$ & $(.000)$ \\
\hline & $r^{2}$ & .194 & .082 & .101 & .175 & .171 \\
\hline \multirow{3}{*}{ Desconfianza/abuso } & Rho Spearman & .370 & .213 & .205 & .455 & .277 \\
\hline & (p-valor) & $(.000)$ & $(.000)$ & $(.000)$ & $(.000)$ & $(.000)$ \\
\hline & $r^{2}$ & .137 & .053 & .042 & .207 & .077 \\
\hline \multirow{3}{*}{ Privación emocional } & Rho Spearman & .326 & .182 & .158 & .411 & .228 \\
\hline & (p-valor) & $(.000)$ & $(.000)$ & $(.000)$ & $(.000)$ & $(.000)$ \\
\hline & $r^{2}$ & .106 & .033 & .025 & .194 & .066 \\
\hline \multirow{3}{*}{ Vulnerabilidad } & Rho Spearman & .285 & .174 & .178 & .326 & .209 \\
\hline & (p-valor) & $(.000)$ & $(.000)$ & $(.000)$ & $(.000)$ & $(.000)$ \\
\hline & $r^{2}$ & .081 & .030 & .032 & .106 & .044 \\
\hline \multirow{3}{*}{ Autosacrificio } & Rho Spearman & .120 & -.012 & .037 & .184 & .158 \\
\hline & (p-valor) & $(.002)$ & $(.753)$ & $(.353)$ & $(.000)$ & $(.000)$ \\
\hline & $r^{2}$ & .081 & .000 & .001 & .034 & .025 \\
\hline \multirow{3}{*}{ Estándares inflexibles 1} & Rho Spearman & .298 & .150 & .175 & .376 & .235 \\
\hline & (p-valor) & $(.000)$ & $(.000)$ & $(.000)$ & $(.000)$ & $(.000)$ \\
\hline & $r^{2}$ & .021 & .023 & .031 & .141 & .055 \\
\hline \multirow{3}{*}{ Estándares inflexibles 2} & Rho Spearman & .146 & .023 & .089 & .179 & .168 \\
\hline & (p-valor) & $(.000)$ & $(.559)$ & $(.024)$ & $(.000)$ & $(.000)$ \\
\hline & $r^{2}$ & .021 & .001 & .008 & .032 & .028 \\
\hline \multirow{3}{*}{ Inhibición emocional } & Rho Spearman & .210 & .050 & .095 & .311 & .195 \\
\hline & (p-valor) & $(.000)$ & $(.208)$ & $(.016)$ & $(.000)$ & $(.000)$ \\
\hline & $r^{2}$ & .044 & .003 & .009 & .097 & .038 \\
\hline \multirow{3}{*}{ Derecho } & Rho Spearman & .332 & .224 & .270 & .298 & .276 \\
\hline & (p-valor) & $(.000)$ & $(.000)$ & $(.000)$ & $(.000)$ & $(.000)$ \\
\hline & $r^{2}$ & .110 & .050 & .073 & .089 & .076 \\
\hline \multirow{3}{*}{ Entrampamiento } & Rho Spearman & .102 & .005 & .064 & .135 & .113 \\
\hline & (p-valor) & $(.010)$ & $(.891)$ & $(.104)$ & $(.001)$ & $(.004)$ \\
\hline & $r^{2}$ & .010 & .000 & .004 & .018 & .013 \\
\hline
\end{tabular}

Nota: $A$ = agresividad; $A F=$ agresividad física; $A V=$ agresividad verbal; $I=$ ira; $H=$ hostilidad; $r^{2}=$ coeficiente de determinación, estimador del tamaño del efecto (Castillo, 2014). 
En la tabla 7 se han comparado las variables de estudio según el sexo. Las diferencias estadísticamente significativas ( $p$-valor <.05) se identificaron en el esquema vulnerabilidad, la variable agresividad y en el componente agresividad física, que fueron más predominantes en los varones, en tanto que en las mujeres se hallaron diferencias estadísticamente significativas en los esquemas de abandono, autosacrificio y entrampamiento.

Adicionalmente, se estimó el tamaño del efecto ( $r$ de Rosenthal) cuyos valores absolutos inferiores al 10 son considerados insignificantes. Cabe precisar que los estimadores de significancia estadística ( $p$-valor) y significancia práctica coinciden, excepto en la variable agresividad.

\section{Tabla 7}

Comparación de las variables esquemas maladaptativos y agresividad, según sexo, en estudiantes de nivel secundario $(N=641)$

\begin{tabular}{|c|c|c|c|c|}
\hline \multirow[b]{2}{*}{ Variables y dimensiones } & \multicolumn{2}{|c|}{ Rango promedio } & \multirow{2}{*}{$\begin{array}{l}\text { Ude Mann-Whitney } \\
\text { (p-valor) }\end{array}$} & \multirow[b]{2}{*}{$r$} \\
\hline & $\begin{array}{l}\text { Varones } \\
(\mathrm{n}=329)\end{array}$ & $\begin{array}{l}\text { Mujeres } \\
(n=312)\end{array}$ & & \\
\hline \multicolumn{5}{|l|}{ Esquemas maladaptativos } \\
\hline Abandono & 302,39 & 338,64 & $45519(.013)$ & $-0,10$ \\
\hline $\begin{array}{l}\text { Insuficiente/autocontrol/ } \\
\text { autodisciplina }\end{array}$ & 320,62 & 321,36 & $51205.5(.960)$ & 0,00 \\
\hline Desconfianza/abuso & 330,08 & 312,39 & $48492(.226)$ & $-0,05$ \\
\hline Privación emocional & 323,05 & 319,05 & $50683,5(.784)$ & $-0,01$ \\
\hline Vulnerabilidad & 340,11 & 302,88 & $45361(.011)$ & $-0,10$ \\
\hline Autosacrificio & 282,66 & 357,36 & $39361(.000)$ & $-0,20$ \\
\hline Estándares inflexibles 1 & 322,73 & 319,36 & $50785(.818)$ & $-0,01$ \\
\hline Estándares inflexibles 2 & 329,44 & 313,00 & $48692(.259)$ & $-0,04$ \\
\hline Inhibición emocional & 317,99 & 323,86 & $50384(.687)$ & $-0,02$ \\
\hline Derecho & 338,63 & 304,28 & $45824,5(.018)$ & $-0,09$ \\
\hline Entrampamiento & 298,70 & 342,15 & $44366,5(.003)$ & $-0,12$ \\
\hline Agresividad & 338,45 & 304,45 & $45879,5(.020)$ & $-0,09$ \\
\hline Agresividad física & 372,97 & 271,72 & $35110,5(.000)$ & $-0,27$ \\
\hline Agresividad verbal & 329,66 & 312,79 & $48623(.248)$ & $-0,05$ \\
\hline Ira & 320,42 & 321,55 & 51 143,5 (.939) & 0,00 \\
\hline Hostilidad & 306,67 & 334,59 & $46853(.056)$ & $-0,08$ \\
\hline
\end{tabular}

Nota: $r=r$ de Rosenthal, estimador del tamaño del efecto (Field, 2013).

Asimismo, se compararon las variables y sus dimensiones según la gestión escolar de la institución educativa. Las diferencias estadísticamente significativas ( $p$-valor <.05), 
en favor de la gestión escolar pública, estuvieron presentes en los esquemas desconfianza, privación emocional, inhibición emocional y en el componente ira (ver tabla 8); en contraste, las diferencias estadísticamente significativas ( $p$-valor <.05), en favor de la gestión escolar privada, se hicieron presentes en los esquemas insuficiente autocontrol/ autodisciplina, derecho y en el componente agresividad verbal. También fue estimado el tamaño del efecto ( $r$ de Rosenthal), cuyos valores absolutos inferiores al .10 son considerados insignificantes.

Cabe mencionar que los estimadores de significancia estadística ( $p$-valor) y significancia práctica ( $r$ de Rosenthal) coinciden, excepto en el esquema desconfianza y el componente agresividad verbal.

Tabla 8

Comparación de las variables esquemas maladaptativos y agresividad, según gestión escolar, en estudiantes de nivel secundario $(N=641)$

\begin{tabular}{|c|c|c|c|c|}
\hline \multirow[b]{2}{*}{ Variables y dimensiones } & \multicolumn{2}{|c|}{ Rango promedio } & \multirow{2}{*}{$\begin{array}{l}\text { Ude Mann-Whitney } \\
\text { (p-valor) }\end{array}$} & \multirow[b]{2}{*}{$r$} \\
\hline & $\begin{array}{l}\text { Público } \\
(n=424)\end{array}$ & $\begin{array}{l}\text { Privado } \\
(\mathrm{n}=217)\end{array}$ & & \\
\hline \multicolumn{5}{|l|}{ Esquemas maladaptativos } \\
\hline Abandono & 318,70 & 325,50 & 45027 (.659) & $-0,02$ \\
\hline $\begin{array}{l}\text { Insuficiente control/ } \\
\text { autodisciplina }\end{array}$ & 306,96 & 348,44 & $40050,5(.007)$ & $-0,11$ \\
\hline Desconfianza/abuso & 332,13 & 299,25 & $41284,5(.033)$ & $-0,08$ \\
\hline Privación emocional & 343,19 & 277,64 & $36595(.000)$ & $-0,17$ \\
\hline Vulnerabilidad & 312,56 & 337,49 & $42425(.106)$ & $-0,06$ \\
\hline Autosacrificio & 312,54 & 337,52 & $42419(.105)$ & $-0,06$ \\
\hline Estándares inflexibles 1 & 327,22 & 308,85 & $43367(.233)$ & $-0,05$ \\
\hline Estándares inflexibles 2 & 326,81 & 309,66 & $43542,5(.265)$ & $-0,04$ \\
\hline Inhibición emocional & 342,02 & 279,93 & $37092,5(.000)$ & $-0,16$ \\
\hline Derecho & 308,00 & 346,40 & $40493(.013)$ & $-0,10$ \\
\hline Entrampamiento & 315,59 & 331,57 & $43710,5(.297)$ & $-0,04$ \\
\hline Agresividad & 323,85 & 315,44 & $44796,5(.586)$ & $-0,02$ \\
\hline Agresividad física & 324,32 & 314,51 & $44596(.525)$ & $-0,03$ \\
\hline Agresividad verbal & 310,08 & 342,35 & $41372(.036)$ & $-0,08$ \\
\hline Ira & 337,05 & 289,65 & $39200,5(.002)$ & $-0,12$ \\
\hline Hostilidad & 318,52 & 325,85 & $44950,5(.634)$ & $-0,02$ \\
\hline
\end{tabular}

Nota: $r=r$ de Rosenthal, estimador del tamaño del efecto (Field, 2013) 


\section{DISCUSIÓN}

La presente investigación se llevó a cabo con estudiantes de cuarto y quinto año de secundaria, pertenecientes a instituciones tanto estatales como particulares de Lima Metropolitana, cuyas edades fluctuaron entre los catorce y dieciocho años. De ello se desprendió que el 51,3\% pertenecían al sexo femenino, en tanto que el 48,7 \% al sexo masculino; a su vez, se obtuvo que el 33,5 \% correspondían por ciento correspondía al cuarto año y el $66,5 \%$ al quinto año de secundaria.

Antes de la evaluación de los datos en función de las hipótesis, se abordó la confiabilidad, de lo cual se obtuvo que más de la mitad de los esquemas maladaptativos tempranos presentaban una confiabilidad ubicada en el nivel moderado; a su vez, la variable agresividad obtuvo un alfa de Cronbach ordinal elevado.

Con relación a la hipótesis general, esta fue aceptada, ya que se hallaron diferencias estadísticamente significativas al correlacionar los esquemas maladaptativos tempranos y la agresividad; es decir, ante un mayor desarrollo de patrones disfuncionales de pensamientos y emociones, el adolescente tendrá una mayor posibilidad de presentar un comportamiento agresivo. Este hallazgo es coherente con resultados previos como el de Chupillón (2019). En este estudio se encontró una correlación positiva y altamente significativa entre los esquemas de abandono, insuficiente autocontrol/ autodisciplina, inhibición emocional y la agresividad controlada e impulsiva; es decir, la adquisición de creencias disfuncionales durante los primeros años de vida permitirá el desarrollo de comportamientos descontrolados o premeditados que tengan como objetivo dañar la figura percibida como amenazadora. Asimismo, en el estudio realizado por Salvatierra (2017), se identificó que los pensamientos disfuncionales tales como privación emocional, abandono, desconfianza/abuso, vulnerabilidad, insuficiente autocontrol/ autodisciplina y derecho guardan relación significativa con la agresividad; en otras palabras, aquellos adolescentes que durante sus primeros años de vida desarrollen la creencia de que las personas más significativas de su entorno no satisfarán sus necesidades emocionales, los lastimarán, engañarán o se desvincularán de su rol protector, desarrollarán dificultades en el control de sus emociones e impulsos.

De la relación encontrada entre ambas variables también se desprende que, a mayor desarrollo de un estilo de crianza saludable durante los primeros años de vida, menor será la posibilidad de presentar comportamientos agresivos durante la adolescencia. Esto podría entenderse a partir de la importancia que representa el desarrollo de una base socioemocional saludable durante la etapa de la niñez e infancia, factor que permitirá la adquisición y desarrollo de conductas autocontroladas y empáticas. Ello es reforzado con investigaciones realizadas por De la Torre-Cruz, García-Linares y Casanova-Arias (2014), así como Arangoitia (2017) y Candela (2017), quienes identificaron que los adolescentes que percibían en sus padres la puesta en práctica de estilos de 
crianza democráticos presentaban menores puntuaciones de agresividad que aquellos estudiantes que describían a sus padres como autoritarios, es decir, a mejor clima sociofamiliar, menor será la posibilidad de adquirir y desarrollar comportamientos agresivos.

Esta relación negativa y significativa también se ha podido observar en un estudio realizado por Arias (2013), quien identificó que los factores familiares tales como los estilos de crianza, modelo de los padres e interacciones sociales tienden a influir en la expresión de conductas agresivas; a su vez, se encontró que el desarrollo de vínculos débiles guarda relación con la adquisición de escasas habilidades empáticas, baja tolerancia a la frustración y egocentrismo.

En lo que respecta a las características de los adolescentes según las variables estudiadas, se percibe un predominio del nivel medio de agresividad. Este resultado coincide con lo identificado por Quijano y Ríos (2015), quienes hallaron niveles medio de agresividad en adolescentes de un colegio nacional en Chiclayo. Asimismo, en los componentes agresividad física, agresividad verbal y hostilidad sobresalió el nivel medio; en tanto que el componente ira obtuvo un predominio del nivel bajo. Resultados similares halló Lozano (2018). De lo expuesto se desprende la importancia de abordar los factores protectores del adolescente con el objetivo de prevenir situaciones de riesgo. El apoyo social generará recursos de afrontamiento, lo que permitirá el desarrollo de un proyecto de vida saludable en los planos tanto psicológico como social (Orcasita y Uribe, 2010).

Con relación a los esquemas maladaptativos tempranos, se percibió predominio de las creencias de privación emocional $(38,2 \%)$ e insuficiente autocontrol/autodisciplina (16,2 \%). Este resultado es respaldado por Flores y Vilca (2017), quienes identificaron que en más de la mitad de su grupo de estudio (adolescentes) predominó el esquema privación emocional. A partir de lo hallado, se expone la importancia de que los cuidadores principales de los niños cubran sus necesidades socioemocionales básicas, ya que la ausencia de figuras permanentes y estables podría generar sentimientos de no pertenencia, inseguridad y desprotección (Seperak, Díaz, Canazas y Shelach, 2018). Puede añadirse a lo ya expuesto que la adquisición de creencias disfuncionales a temprana edad generará entre otras consecuencias, dificultades en el control de las emociones, impulsos y escasa disciplina (Castrillón et al., 2005).

En lo que respecta a la correlación entre los componentes de ambas variables, se observó la existencia de una relación significativa entre todos los esquemas maladaptativos tempranos y la agresividad verbal, con excepción de los esquemas autosacrificio y entrampamiento; es decir, el contexto social durante los primeros años de vida, ya sea a través de la familia, colegio u otros, ejerce gran influencia en el desarrollo de la agresividad (Londoño, 2010). Asimismo, con relación al componente agresividad física, los resultados arrojaron una correlación significativa en todos los esquemas, con excepción de los estándares inflexibles 2 , inhibición emocional y entrampamiento. Puede añadirse 
a lo ya mencionado que todos los esquemas maladaptativos tempranos se correlacionan significativamente con los componentes hostilidad e ira; en otras palabras, la exposición a factores de riesgo durante los primeros años de vida, tales como padres disfuncionales, relación deteriorada entre progenitores o violencia intrafamiliar reforzará la adquisición de conductas que tengan como objetivo causar daño a los demás. Cabe señalar que la presencia de conductas altamente agresivas durante la niñez pronosticará el desarrollo de la agresividad en la adolescencia (Serrano, 2003); adjunto a ello, posiblemente se observe la adquisición y desarrollo de adicciones (Matalinares et al., 2013), escasas habilidades de integración social (Montoya, 2014), baja autoestima (León, 2017) y aumento de creencias irracionales (Requejo, 2018).

En lo que respecta al análisis diferencial de ambas variables, esquemas maladaptativos tempranos y agresividad, según el sexo, se obtuvieron diferencias significativas. En la variable agresividad se percibe que los varones tienden a ser más agresivos que las mujeres. Estos resultados se refuerzan con los obtenidos por Carbajal y Jaramillo (2015), quienes identificaron que los adolescentes varones presentan mayores niveles de agresividad que las mujeres. Ello coincide con lo señalado por Castrillón y Vieco (2002), quienes también plantean que la agresividad es mayor en los varones, ya que a las mujeres se les ha permitido socialmente emplear estrategias emocionales como forma de expresión de sus necesidades, frustración, discrepancias y expectativas, en tanto que a los varones se les ha inhibido emocionalmente, llevándolos a seguir estereotipos de género en los que el poder y vitalidad guardan estrecha relación con la agresividad. Asimismo, los varones han presentado mayores niveles de agresividad física que las mujeres, lo que coincide con lo planteado por Matalinares et al. (2013), quienes refieren que en diversos estudios se ha encontrado que la expresión de agresividad de los varones guarda relación con aspectos biológicos de género y del genotipo. Por su parte, Castrillón y Vieco (2002) expresan que la agresión física tiende a ser mayor en los varones, ya que, debido a estrategias genéticas, desde épocas remotas se ha exigido al varón ejercer un rol de protección y superioridad física.

Por otro lado, en lo que respecta a la variable esquemas maladaptativos tempranos, las mujeres tienden a presentar mayor prevalencia de los esquemas abandono, autosacrificio y entrampamiento, en tanto que en los varones prevalece los esquemas de vulnerabilidad y derecho. Estos resultados guardan relación con lo hallado por Salvatierra (2017), quien identificó que las adolescentes pertenecientes al género femenino tienden a priorizar las necesidades de los demás antes que las suyas; a su vez, tanto Chupillón (2019) como Gantiva et al. (2009) hallaron el predominio de los esquemas maladaptativos tempranos en mujeres. Torres y Pérez (2016) determinaron que en las adolescentes embarazadas suele perpetuarse el esquema de abandono; no obstante, Agudelo, Casadiegos y Sánchez (2007) identificaron, en estudiantes universitarios, que en los varones tendía a prevalecer el desarrollo de esquemas tales como autosacrificio, inhibición emocional 
y derecho, este último esquema similar a lo identificado en la presente investigación. Esto significaría que, tanto para el varón como para la mujer, resulta fundamental vivir un desarrollo saludable durante los primeros años de vida, ya que ambos géneros son vulnerables a perpetuar pensamientos o emociones disfuncionales.

Con relación a la gestión escolar, se aprecia que en los colegios públicos tiende a prevalecer la ira, en tanto que en los privados predomina la agresividad verbal; es decir, en aquellas instituciones en las que se percibe un nivel socioeconómico con mayores recursos, los adolescentes tienden a imponer o expresar de manera impulsiva sus ideas y pensamientos, en tanto que los estudiantes de colegios nacionales suelen experimentar sentimientos de haber sido dañados o vulnerados. Estos resultados son reforzados con los hallados por Díaz (2017), quien encontró mayores niveles de agresividad física en zonas rurales en comparación a las urbanas. A su vez, Matalinares et al. (2012) expusieron que los adolescentes pertenecientes a la costa serían más vulnerables a desarrollar conductas de riesgo.

Con relación a los esquemas maladaptativos tempranos, en los colegios públicos prevalecieron los esquemas de desconfianza, privación e inhibición emocional, mientras que en los privados sobresalieron las creencias, insuficiente autocontrol/autodisciplina y derecho; en otras palabras, los estudiantes de colegios privados han desarrollado la creencia de que son superiores a los demás, razón por la cual gozan de derechos y privilegios carentes de límites y normas (Castrillón et al., 2005), en tanto que los estudiantes de colegios públicos tienden a inhibir la expresión de sus emociones por temor a ser heridos por los demás. La carencia de un apoyo socioemocional adecuado a temprana edad ha generado en ellos inseguridad, necesidad de pertenencia, aprobación y protección. Resultados similares fueron hallados por Salvatierra (2017), quien identificó, en estudiantes de instituciones nacionales, el desarrollo de pensamientos relacionados con el aprovechamiento de los demás hacia ellos, razón por la cual optan por suprimir la expresión de sus necesidades o sentimientos.

En síntesis, la presente investigación resalta la relación positiva y significativa entre los esquemas maladaptativos tempranos y la agresividad en adolescentes. De ello se desprende la importancia de promover una niñez, infancia y adolescencia saludable, ya que ello permitirá reducir los factores de riesgo a través de la adquisición y desarrollo de factores protectores tanto en el ámbito personal como en el social.

Asimismo, diversos estudios han mostrado que la agresividad no solo se relaciona con los esquemas maladaptativos tempranos sino también con otras variables que vulneran el desarrollo del estudiante, por ello resulta importante crear programas preventivos promocionales con padres e hijos con el objetivo de conducir adecuadamente los pensamientos y emociones del adolescente. 


\section{CONCLUSIONES}

Existe una relación positiva y significativa entre los esquemas maladaptativos tempranos y la agresividad en estudiantes de secundaria de Lima Metropolitana.

Existe una relación directa y significativa entre los esquemas maladaptativos tempranos y las subescalas de la agresividad, con excepción de las correlaciones agresividad verbal con los esquemas autosacrificio y entrampamiento, así como la agresividad física con los esquemas estándares inflexibles 2, inhibición emocional y entrampamiento en estudiantes de secundaria de Lima Metropolitana.

En un nivel descriptivo, los esquemas maladaptativos tempranos que prevalecieron fueron privación emocional e insuficiente autocontrol/autodisciplina. En la escala agresividad y sus subescalas, predominó la categoría medio, con excepción de la ira, en la cual resaltó el nivel bajo.

Con relación al sexo, se obtuvieron diferencias significativas en ambas variables: los varones tienden a ser más agresivos que las mujeres, en tanto que en las mujeres prevaleció el desarrollo de esquemas tales como abandono, autosacrificio y entrampamiento.

En lo que respecta a la gestión escolar, en las instituciones públicas predominó la ira, desconfianza, privación emocional e inhibición emocional, en tanto que en los colegios privados prevaleció la agresión verbal y los esquemas insuficiente autocontrol/ autodisciplina y derecho.

\section{REFERENCIAS}

Agip, J. (2018). Agresividad en estudiantes de cuarto año de secundaria de una institución educativa pública del distrito de Puente Piedra (tesis de pregrado). Lima: Universidad Inca Garcilaso de la Vega.

Agudelo, D., Casadiegos, C. y Sánchez, D. (2007). Relación entre esquemas maladaptativos tempranos y características de ansiedad y depresión en estudiantes universitarios. Universitas Psychologica, 8(1), 87-103.

Arangoitia, A. (2017). Clima social familiar y agresividad en adolescentes de 3ro, 4to y 5to de secundaria de la Institución Educativa Parroquial San Columbano (tesis de pregrado). Lima: Universidad César Vallejo.

Arias, W. (2013). Agresividad y violencia en la adolescencia: la importancia de la familia. Avances en psicología, 21(1), 23-34.

Buss A. y Perry, M. (1992). The aggression Questionnaire. Journal of Personality and Social Psychology. Vol. 63, Nº 3, 452-459.

Candela, V. (2017). Patrones de comportamiento parental y agresividad en adolescentes del distrito de San Vicente, Cañete (tesis de maestría). Lima: Universidad Nacional Federico Villarreal. 
Carbajal, M. y Jaramillo, J. (2015). Conductas agresivas de los alumnos del primer año nivel secundaria Institución Educativa Técnico Industrial Pedro E. Paulet (tesis de pregrado). Huacho: Universidad Nacional José Faustino Sánchez Carrión.

Castrillón, D. y Vieco, F. (2002). Actitudes justificativas del comportamiento agresivo y violento en estudiantes universitarios de la ciudad de Medellín, Colombia. Rev. Fac. Salud Pública, 20(2), 51-66.

Castrillón, D., Chaves, L., Ferrer, A., Londoño, N., Maestre, K., Marín, C., y Schnitter, M. (2005). Validación del Young Schema Questionnaire Long Form- Second Edition (YSQ - L2) en población colombiana. Revista Latinoamericana de Psicología, 37(3), 541-560.

Chupillón, M. (2019). Esquemas maladaptativos tempranos y agresividad premeditadaimpulsiva en estudiantes de una institución educativa de Chiclayo (tesis de pregrado). Lima: Universidad Señor de Sipán.

De la Torre-Cruz, M., García-Linares, M. y Casanova-Arias, P. (2014). Relaciones entre estilos educativos parentales y agresividad en adolescentes. Electronic Journal of Research in Psychology, 12(1), 147-170.

Díaz, F. (2017). Niveles de agresividad en adolescentes entre 14 y 16 años, en zona rural y urbana del distrito de Jaén-Cajamarca (tesis de pregrado). Cajamarca: Universidad Privada del Norte.

Domínguez-Lara, S. (2018). Fiabilidad y alfa ordinal. Actas Urológicas Españolas, 42(2), 140-141. doi: dx.doi.org/10.1016/j.acuro.2017.07.002

Field, A. (2013) Discovering statistics using IBM SPSS Statistics: and sex and drugs and rock ' $n$ ' roll (4th ed.). London: Sage.

Flores, M. y Vilca, M. (2017). Esquemas desadaptativos tempranos en adolescentes con problemas de conducta (tesis de pregrado). Arequipa: Universidad Nacional de San Agustín, Arequipa.

Gadermann, A. M., Guhn, M. y Zumbo, Br. (2012). Estimating ordinal reliability for Likerttype and ordinal item response data: A conceptual, empirical, and practical guide. Practical Assessment, Research \& Evaluation, 17(3). Recuperado de http:// pareonline.net/getvn.asp? $v=17 \& n=3$

Gantiva, C., Bello, A., Vanegas, E. y Sastoque, Y. (2009). Historia de maltrato físico en la infancia y esquemas maladaptativos tempranos en estudiantes universitarios. Acta Colombiana Psicológica, 12(2), 127-134. Recuperado de http://www.scielo. org.co/scielo.php?script=sci_arttext\&pid=S0123-91552009000200012

Gantiva, C., Bello, J., Vanegas, E. y Sastoque, Y. (2010). Relación entre el consumo excesivo de alcohol y esquemas maladaptativos tempranos en estudiantes universitarios. RevistaColombianadePsiquiatría,39(2),362-374.Recuperadodehttp://www.scielo. org.co/scielo.php?script=sci_arttext\&pid=S0034-74502010000200010\&lng=en \&tlng=es 
Hogan, Th. (2015). Pruebas psicológicas. México, D. F.: Manual Moderno.

Inglés, C., Torregrosa, M., García- Fernández, J., Martínez-Monteagudo, M., Estévez, E. y Delgado, B. (2014). Conducta agresiva e inteligencia emocional en la adolescencia. European Journal of Education and Psychology, 7(1), 29-41. Recuperado de file://C:/Users/ACO/Downloads/97-1-122-1-10-20151029.pdf

Instituto Nacional de Estadística e Informática (2019). Estado de la niñez y adolescencia. [Encuesta Nacional de Hogares]. Recuperado de https://www1.inei.gob.pe/ media/MenuRecursivo/boletines/boletin_ninez.pdf

León, M. (2017). Niveles de autoestima y niveles de agresividad en niños y adolescentes evaluados por violencia familiar en la división de Psicología Forense - DEREJCRI PNP (tesis de maestría). Lima: Universidad Inca Garcilaso de la Vega.

León, K. y Sucari, Cl. (2012). Adaptación del cuestionario de esquemas de Young en adolescentes en adolescentes de dos distritos de Lima Sur (tesis de pregrado). Lima: Universidad Femenina del Sagrado Corazón.

Londoño (2010). Agresividad en niños y niñas, una mirada desde la Psicología Dinámica. Revista virtual Universidad Católica del Norte, 31, 274-293. Recuperado de http:// revistavirtual.ucn.edu.co/index.php/RevistaUCN/article/view/45

Lozano, Y. (2018). Conductas agresivas en alumnos de cuarto y quinto de educación secundaria de una institución educativa pública del distrito de Villa María del Triunfo (tesis de pregrado). Lima: Universidad Inca Garcilaso de la Vega.

Luna, B. (2017). Agresividad en estudiantes de secundaria de la Institución Educativa Pública 3048 del distrito de Independencia (tesis de pregrado). Lima: Universidad Inca Garcilaso de la Vega.

Manterola C. y Otzen, T. (2017). Los sesgos en investigación clínica. International Journal of Morphology, 33(3), 1156-1164. doi: dx.doi.org/10.4067/S0717-950 22015000300056

Matalinares, M., Arenas, C., Sotelo, L., Díaz, G., Dioses, A., Yaringaño, J., Muratta, R., Pareja, C. y Tipacti, R. (2010). Clima familiar y agresividad en estudiantes de secundaria de Lima Metropolitana. Revista de Investigación en Psicología, 13(1), 109-128.

Matalinares, M., Yaringaño, J., Uceda, J., Fernández, E., Huari, Y., Campos, A. y Villavicencio, N. (2012). Estudio psicométrico de la versión española del cuestionario de agresión de Buss y Perry. Revista de Investigación en Psicología, 21(1), 147-161.

Matalinares, M., Arenas, C., Díaz, G. y Dioses, A. (2014). Adicción a la internet y agresividad en estudiantes de secundaria del Perú. Revista de Investigación en Psicología, 16(1), 75-93.

Minedu (2018). Censo escolar 2018. Lima: Estadísticas de la Calidad Educativa. 
Molina, A. (2018). Agresividad en estudiantes de secundaria de una Institución Educativa Estatal del distrito de San Miguel (tesis de pregrado). Lima: Universidad Inca Garcilaso de la Vega.

Montoya, R. (2014). Agresividad premeditada - impulsividad y acoso escolar en adolescentes de secundaria. Revista de Investigación Altoandina, 16(1), 139-148.

Orcacita, L. y Uribe, A. (2010). La importancia del apoyo social en el bienestar de los adolescentes. Psychologia: Avances de la Disciplina, 4(2), 69-82.

Pérez, L. y Torres, A. (2016). Esquemas maladaptativos en madres adolescentes residentes en un centro de atención en Lima (tesis de pregrado). Lima: Universidad Femenina del Sagrado Corazón.

Quijano, S. y Ríos, M. (2015). Agresividad en adolescentes de educación secundaria de una Institución Educativa Nacional La Victoria, Chiclayo (tesis de pregrado). Chiclayo: Universidad Católica Santo Toribio de Mogrovejo.

Requejo, A. (2018). Creencias irracionales y agresividad premeditada e impulsiva en adolescentes de una Institución Educativa Pública del distrito de San Martín de Porres (tesis de pregrado). Lima: Universidad César Vallejo.

Rice, P. (2000). Adolescencia: desarrollo, relaciones y cultura. Madrid: Prentice Hall.

Rodríguez, E. (2009). La terapia centrada en esquemas de Jeffrey Young. Avances en psicología, 17(1), 59-74.

Romano, J. L., Kromrey, J. D., Owens, C.M. y Scott, H.M. (2011). Confidence interval methods for coefficient alpha on the basis of discrete, ordinal response items: Which one, if any, is the best? Journal of Experimental Education, 79, 382-403. doi: $10.1080 / 00220973.2010 .510859$

Salvatierra, N. (2017). Esquemas maladaptativos tempranos y agresividad en estudiantes de secundaria de instituciones educativas públicas del distrito de Carabayllo (tesis de pregrado). Lima: Universidad César Vallejo.

Sánchez, H. y Reyes, C. (2009). Metodología y diseños en la investigación científica. Lima: Visión Universitaria.

Sánchez, A., Andrade, P. y Lucio, M. (2019). Esquemas desadaptativos tempranos y ansiedad en escolares de México. Revista de Psicología Clínica con Niños y Adolescentes, 6(2), 15-21.

Salgado (2018). Manual de investigación. Teoría y práctica para hacer la tesis según la metodología cuantitativa. Lima: Fondo Editorial de la Universidad Marcelino Champagnat.

Seperak, R., Díaz, L., Canazas, M. y Shelach, S. (2018). Esquemas mentales desadaptativos según la composición familiar en adolescentes arequipeños de escuelas públicas. Revista de Investigación en Psicología, 21(2), 236-254. 
Shaffer, D. y Kipp, K. (2007). Psicología del desarrollo. Infancia y adolescencia. México, D. F.: Thomson.

Serrano, E. (2003). Agresividad infantil. Madrid: Pirámide.

Turner, H.J. (2015). A performance evaluation of confidence intervals for ordinal coefficient alpha. Doctor of Philosophy. Texas: University of North Texas.

Young, J. E. (1990). Cognitive therapy for personality disorders: a schema-focused approach. Sarasota: Professional Resourse Exchange, Inc. 